\title{
DNA-nanodiamond interactions influence on fluorescence of nanodiamonds
}

\author{
E. N. Vervald ${ }^{1}$, K. A. Laptinskiy ${ }^{1}$, I. I. Vlasov ${ }^{2,3}$, O. A. Shenderova ${ }^{4}$, T. A. Dolenko ${ }^{1,3}$ \\ ${ }^{1}$ Lomonosov Moscow State University, Moscow, Russia \\ ${ }^{2}$ A. M. Prokhorov General Physics Institute, Russian Academy of Science, Moscow, Russia \\ ${ }^{3}$ National Research Nuclear University MEPhI, Moscow, Russia \\ ${ }^{4}$ Adamas Nanotechnologies, Inc, Raleigh, NC, USA \\ en.khusainova@physics.msu.ru
}

PACS 87.64.-t, 87.64.kv, 87.64.kp, 87.64.K-

DOI 10.17586/2220-8054-2018-9-1-64-66

\begin{abstract}
In this study, the interaction between DNA and the surface of detonation nanodiamonds and nanodiamonds with NV centers is investigated, and the quantitative parameters of this interaction are calculated. The influence of interaction of DNA with nanodiamonds on the fluorescent properties of nanodiamonds is established. A correlation was found between the efficiency of DNA interaction with the surface of the detonation nanodiamonds and the changes of their fluorescence: the more DNA bonds with the nanodiamond surface groups - the stronger the fluorescence of detonation nanodiamonds increases in water
\end{abstract}

Keywords: nanodiamonds, DNA, interactions, fluorescence.

Received: 20 June 2017

Revised: 20 September 2017

\section{Introduction}

Unique properties of nanodiamonds (NDs) such as nontoxicity, biocompatibility, photostability, ability to be covered with different surface groups allow using them as theranostic agents. There are many publications where it was shown that NDs can successfully be used as fluorescent biomarkers [1], platform for drug delivery [2], adsorbents of heavy metals, viruses and proteins [3,4]. However, to understand all the possibilities for NDs applications in biomedicine it is important to study their interactions with biomacromolecules.

In this study, the interactions of detonation nanodiamonds (DNDs) and nanodiamonds with NV centers (NDNV) and calf thymus DNA molecules in water were investigated.

\section{Materials and methods}

The DNDs functionalized with carboxyl groups with sizes of $5 \mathrm{~nm}$ and $10 \mathrm{~nm}$, and ND-NV with size of $100 \mathrm{~nm}$ with polyfunctional surfaces were used in this study. The synthesis of the samples is described in [5,6]. We note here that $10 \mathrm{~nm}$ DNDs and $5 \mathrm{~nm}$ DNDs were treated differently, as the results of which DNDs $5 \mathrm{~nm}$ were obtained with much more intense fluorescence (FL) due to production of tiny carbon dots on the DNDs surface [7]. Deoxyribonucleic acid sodium salt from calf thymus (Sigma-Aldrich) was used as a DNA sample.

The studies of the interactions of DNA and NDs in aqueous suspensions were carried out using laser Raman spectroscopy (RS) and fluorescence spectroscopy. The Raman spectra and FL spectra of the samples were obtained using a laser spectrometer, as described in $[5,6]$.

\section{Results and discussion}

\subsection{Interaction of DNA chains and the nanodiamond surface}

To study the DNA interaction with the NDs the initial aqueous suspensions of DNDs and ND-NV with concentrations of 2 and $0.2 \mathrm{~g} / \mathrm{L}$, respectively, and the aqueous solutions of DNA with concentration of $13 \mathrm{~g} / \mathrm{L}$ were prepared. They were mixed with each other in the ratio 1:1. After $2 \mathrm{~h}$ of interactions and 40 min of centrifugation (13000 rpm) the supernatant was separated from the precipitate.

The change in the concentrations of DNA in the initial mixture and in the supernatant allowed to determine the amount of DNA bonded with the NDs. The DNA concentration was measured using intensity of the RS marker lines of the complementary pair of guanine $(\mathrm{G})$ and cytosine $(\mathrm{C})$ (Fig. 2 in [8]). It is known that studied DNA consists of $42 \%$ of pairs of G-C. Using these facts and the measured changes in the $\mathrm{G}-\mathrm{C}$ concentration, the total change in the DNA concentration in the solution after bonding was obtained. The estimations showed that $38 \%$ of the initial number of DNA molecules bonded with the $5 \mathrm{~nm}$ DNDs, $34 \%$ - with the $10 \mathrm{~nm}$ DNDs, $20 \%$ - with the ND-NV. The interaction parameter $(P)$ of each NDs with DNA was calculated. $P$ is the ratio of the amount 
of bonded substance by weight to the ND's surface area. The hydrodynamic radii, obtained by dynamic light scattering method, were used to calculate the surface area of NDs in suspensions. According to [9], the fraction of micropores is less than $1 \%$, which is why we treat our particles as spheres. The obtained results of surface areas are the following: for DND $10 \mathrm{~nm}-17.1 \cdot 10^{-2} \mathrm{~m}^{2}$, for DND $5 \mathrm{~nm}-34.3 \cdot 10^{-2} \mathrm{~m}^{2}$, for ND-NV $-2 \cdot 10^{-2} \mathrm{~m}^{2}$.

The following values were obtained: for DND $5 \mathrm{~nm} P=3.6 \mathrm{mg} / \mathrm{m}^{2}$, for DND $10 \mathrm{~nm} P=6.5 \mathrm{mg} / \mathrm{m}^{2}$, for ND-NV $P=370 \mathrm{mg} / \mathrm{m}^{2}$. Thus, the ND-NV has the highest $P$. In all likelihood, the polyfunctional surface of ND-NV interacts with DNA strands more strongly than with $\mathrm{COOH}$ groups. For the DND $5 \mathrm{~nm}, P$ is the smallest. Apparently, this is due to the presence of carbon dots on the DND $5 \mathrm{~nm}$ surface (see p. 2), which weakly interact with the DNA molecules.

\subsection{The influence of DNA-NDs interaction on ND fluorescence}

The initial aqueous suspensions of DNDs and ND-NV were prepared with concentrations of 1 and $0.06 \mathrm{~g} / \mathrm{L}$, respectively, so as the initial aqueous solutions of DNA with concentrations of 6.3 and $3.75 \mathrm{~g} / \mathrm{L}$. The initial suspensions of NDs and DNA were mixed with each other in the ratio 1:1. The FL and Raman spectra of the aqueous ND suspensions, the solutions of DNA and the mixtures of (NDs + DNA) were obtained. As one can see from Fig. 1, the spectra contain water Raman valence band and broad band of FL in the range from $420 \mathrm{~nm}$ to $750 \mathrm{~nm}$ (a) and from $540 \mathrm{~nm}$ to $850 \mathrm{~nm}$ (b).
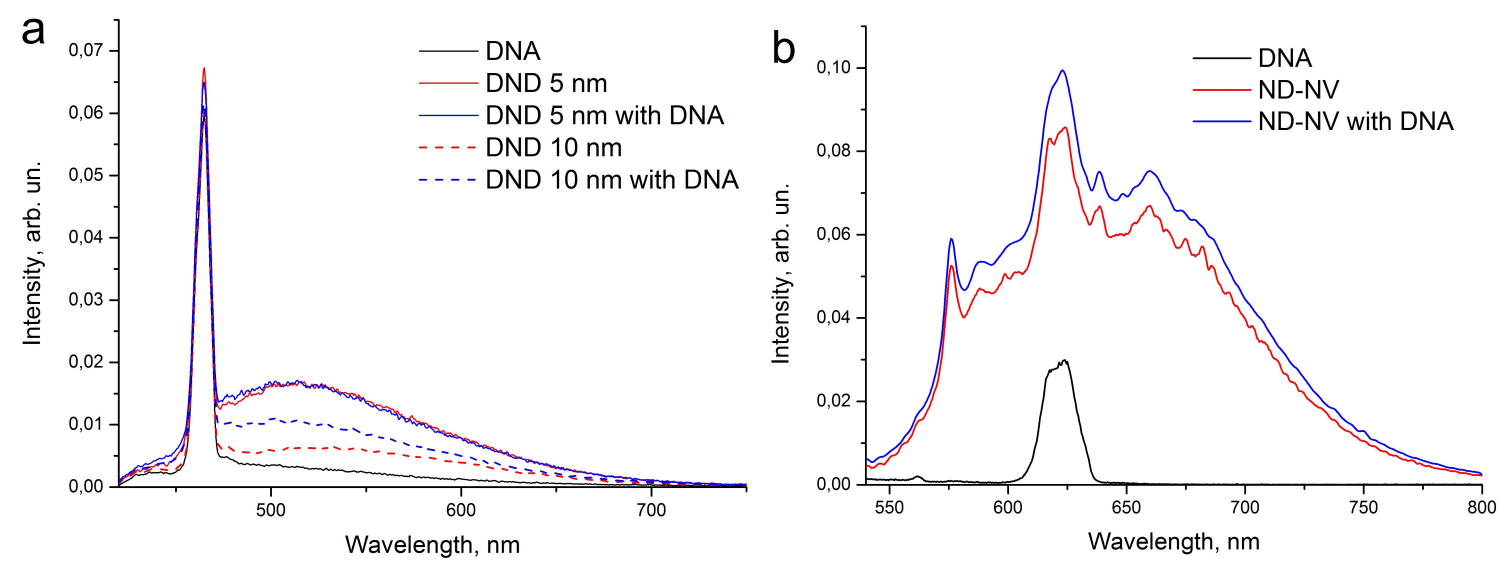

FIG. 1. Raman and fluorescence spectra of DNA, NDs and NDs with DNA mixtures: (a) DND $5 \mathrm{~nm}$ and DND $10 \mathrm{~nm}$, concentration of DNA is $3.15 \mathrm{~g} / \mathrm{L}, \lambda_{e x}=405 \mathrm{~nm}$; (b) NDNV, concentration of DNA is $1.875 \mathrm{~g} / \mathrm{L}, \lambda_{e x}=514.5 \mathrm{~nm}$

It was found that in all samples (NDs + DNA) the FL intensity is not reduced compared with that of NDs in water (Fig. 1). The parameter $F_{0}$, which equals to the ratio of integral FL intensity to integral intensity of water valence band [5], was calculated for each spectrum for quantitative estimations of change in FL of NDs. The calculations showed that as a result of interaction between DNA and NDs, the parameter $F_{0}$ for DND $5 \mathrm{~nm}$ almost did not change $\left(\Delta F_{0}=(1 \pm 4) \%\right), F_{0}$ for DND $10 \mathrm{~nm}$ increased by $(49 \pm 4) \%, F_{0}$ for ND-NV increased by $(15 \pm 4) \%$. The presented results are averaged over 5 experiments.

Thus, there is the correlation between the efficiency of DNA and DNDs interactions and the change in their FL. The higher $P$ - the more change in FL: $P$ for $10 \mathrm{~nm}$ DND is almost 2 times more than that of $5 \mathrm{~nm}$ DND. We explain this by the fact that DNA "shields" surface defects of ND (which cause ND FL [10]) from water molecules which usually quench FL.

Despite the highest $P$ of the ND-NV, the change in its FL is insignificant. From these data, it is clear that the surface defects are responsible for FL of DNDs [10], but the main sources of ND-NV FL are color centers, which are inside the volume, not on the surface. According to [11] the NV centers react to significant interactions of the surface groups with environment up to depth of $20 \mathrm{~nm}$ from the surface. Apparently, the interactions of ND-NV $100 \mathrm{~nm}$ with DNA are not strong enough to change FL of NV centers significantly and therefore to change the FL of ND-NV.

\section{Conclusion}

The significant interactions of DNA molecules with DNDs of $5 \mathrm{~nm}$ and $10 \mathrm{~nm}$ and with ND-NV of $100 \mathrm{~nm}$ have been demonstrated, the parameter of these interactions $P$ for each ND was calculated. 
The influence of interactions of NDs with DNA on the NDs FL properties was observed. The correlation between the efficiency of DNDs-DNA interactions - the parameter $P$ - and the change in FL of DNDs were found: the more DNDs $P$ - the more increase of FL intensity. Despite the highest $P$ of ND-NV, the change of its FL is minor, since the surface defects play small role in the FL of ND-NV.

\section{Acknowledgements}

This study has been performed with support of the grant of Russian Science Foundation project No. 17-1201481 (E.N.V., K.A.L., T.A.D. - planning and conducting of the experiment, discussion of physical results) and was partially supported by the MEPhI Academic Excellence Project contract Nos. 02.a03.21.0005, 27.08.2013 (I.I.V. - discussion of results).

\section{References}

[1] Mochalin V., Shenderova O., Ho D., Gogotsi Y. The properties and applications of nanodiamonds. Nat. Nanotechnol., 2011,7 (1), P. 11-23.

[2] Chen M., Pierstorff E.D., et al. Nanodiamond-mediated delivery of water-insoluble therapeutics. ASC Nano, 2009,7 (3), P. $2012-2022$.

[3] Giammarco J., Mochalin V.N., Haeckel J., Gogotsi Y. The adsorption of tetracycline and vancomycin onto nanodiamond with controlled release. J. Colloid Interface Sci., 2016, 468, P. 253-261.

[4] Girard H., Pager V., Simic V., Arnault J.-C. Peptide nucleic acid-nanodiamonds: Covalent and stable conjugates for DNA targeting. RSC Adv., 2014, 4, P. 3566-3572.

[5] Vervald A., Burikov S., et al. Relationship between fluorescent and vibronic properties of detonation nanodiamonds and strength of hydrogen bonds in suspensions. J. Phys. Chem. C, 2016, 120, P. 19375-19383.

[6] Laptinskiy K., Burikov S., et al. Monitoring of nanodiamonds in human urine using artificial neural networks. Phys. Status Solidi A, 2016, 213 (10), P. 2614-2622.

[7] Shenderova O., Hens S., et al. Carbon dot-decorated nanodiamonds. Part. Part. Syst. Char., 2014,31 (5), P. $580-590$.

[8] Laptinskiy K., Burikov S., Dolenko T. Improvement of reliability of molecular DNA computing: solution of inverse problem of Raman spectroscopy using artificial neural networks. Laser Physics, 2017, 27 (2), P. 025203(8).

[9] Kachevskii S., Golubina E., Lokteva E., Lunin V. Palladium on ultradisperse diamond and activated carbon: the relation between structure and activity in hydrodechlorination. Russian J. Phys. Chem. A, 2007, 81 (6), P. 866-873.

[10] Xiao J., Jun P., Li L., Yang G. Fluorescence origin of nanodiamonds. J. Phys. Chem. C, 2015, 11, P. $2239-2248$.

[11] Petrakova V., Benson V., et al. Imaging of transfection and intracellular release of intact, non-labeled DNA using fluorescent nanodiamonds. Nanoscale, 2016, 8 (23), P. 12002-12012. 